\title{
Stabilization of Gold Nanospheres and Nanorods in Diblock Copolymers of Styrene and Vinylpyridine
}

\author{
Ya. I. Derikov ${ }^{a, *}$, C. Abetz ${ }^{b}$, G. A. Shandryuk ${ }^{a}$, R. V. Talroze ${ }^{a}$, A. A. Ezhov ${ }^{a, c}$, \\ V. Abetz ${ }^{b, d}$, Ya. V. Kudryavtsev ${ }^{a, e}$, and M. A. Osipov ${ }^{a, f}$ \\ ${ }^{a}$ Topchiev Institute of Petrochemical Synthesis, Russian Academy of Sciences, Moscow, 119991 Russia \\ ${ }^{b}$ Helmholtz-Zentrum Geesthacht, Institute of Polymer Research, \\ Max-Planck-Strasse 1, 21502 Geesthacht, Germany \\ ${ }^{c}$ Faculty of Physics, Moscow State University, Moscow, 119991 Russia \\ ${ }^{d}$ Institute of Physical Chemistry, University of Hamburg, \\ Martin-Luther-King-Platz 6, 20146 Hamburg, Germany \\ ${ }^{e}$ Frumkin Institute of Physical Chemistry and Electrochemistry, Russian Academy of Sciences, \\ Moscow, 119991 Russia \\ ${ }^{f}$ Department of Mathematics and Statistics, University of Strathclyde, \\ Glasgow G1 1XH, Scotland, United Kingdom \\ *e-mail: derikov@ips.ac.ru \\ Received November 15, 2017
}

\begin{abstract}
Composites based on microphase-separated diblock copolymers of styrene with vinylpyridine and gold nanoparticles, in which the block copolymers play simultaneously both the role of a stabilizer preventing the aggregation of nanoparticles and a matrix providing their selective arrangement within domains of the predefined type, are obtained for the first time. The suggested approach implies no use of additional stabilizers and is efficient for both initially organic dispersed small $(\sim 5 \mathrm{~nm})$ spheres and of larger $(9 \times$ $30 \mathrm{~nm}$ ) rodlike nanoparticles. Nanospheres are introduced into the composites via replacement of a labile stabilizer used in synthesis with the diblock copolymer, while nanorods synthesized in aqueous media by the two-stage method are incorporated via phase transfer mediated by the diblock copolymer. Films capable of microphase separation by the formation of vinylpyridine cylindrical domains with a diameter of tens nanometers are spin-coated from the obtained composites. The content of nanoparticles in the composites is $5 \mathrm{wt} \%$; they are dispersed and arranged predominantly within vinylpyridine domains, with nanospheres being localized near domain boundaries, while nanorods are mainly oriented perpendicularly to the axis of cylinders.
\end{abstract}

DOI: $10.1134 / \mathrm{S} 1811238218010034$

\section{INTRODUCTION}

The ability of block copolymers to form nanostructures with diverse morphologies and periodicities is well studied [1]. Presently, various methods of controlling the structure of thin block copolymer films are practically brought up to the technological level $[2,3]$. The development of synthetic approaches [4] and the extensions of application of block copolymers to lithography [5], photovoltaics [6], and preparations of membranes [7] and other functional materials demonstrate achievements in this field and, simultaneously, intrinsic polymer restrictions [8]. A potential way to improve the properties of materials based on block copolymers is to create hybrid composites with nanoparticles [9-11], which are also capable of selforganization to highly ordered structures [12]. Depending on their chemical nature, nanoparticles may substantially improve mechanical, barrier, elec- tric, optical, and other characteristics of matrix polymers without sacrificing their good processibility [13, 14]. Thus, block copolymers doped with noble-metal nanoparticles are regarded as promising hybrid materials combining plasmonic, catalytic, and other functional properties of nanoparticles with their periodic spatial arrangement in copolymer domains occurring as a result of microphase separation $[15,16]$. Nanoparticles appropriate for this purpose should be stable against aggregation, miscible with at least one of the copolymer blocks, and similar to domains of the formed microstructure in size and shape.

Of special interest are anisometric nanoparticles sensitive to external fields and able to significantly enhance the anisotropic properties of composites based on lamellar and hexagonal microstructures typical of block copolymers. Recent theoretical investigations revealed that the models of liquid-crystalline 
ordering of nanoparticles in the periodic structure of domains of a block copolymer are promising [17-19] and their main results agree well with the data of mesoscopic simulations [20]. At the same time, there are only a few experimental studies of nanorod-filled block copolymers, especially for gold nanorods [2124]. Although there are proven synthetic methodologies making it possible to obtain $\mathrm{Au}$ nanorods with high yields and desired aspect ratios in the range of 1.5-5.0 [25-27], their applicability is limited because of the hydrophilicity of nanorods and the need to make them organophilic before their incorporation into polymer matrices, except the case where the polymer host itself is hydrophilic.

In a recent paper [28], a fast and suitable method of transferring gold nanorods from aqueous to organic media using the block copolymers of styrene with 2- or 4-vinylpyridine (PS-P-2-VP, PS-P-4-VP) as phasetransfer agents was described. This method is based on the known [29-31] ability of the gold surface to selectively interact with a vinylpyridine block, resulting from the presence of a pyridine ring in the polymer repeating unit. It is assumed [31] that these blocks are adsorbed on nanorods, enhancing interactions with the surface owing to a large amount of contacts.

In the present paper, we will show that the blockcopolymer composite with nanorods formed in the phase-transfer process may be used to prepare thin films and to transfer them to the microphase-separated state. It should be noted that the known examples of incorporating Au nanorods into block copolymers imply the use of either an etched template [23] or additional stabilizers, such as relatively short chains of PEG [21], PS [22], or P-2-VP [24] with terminal thiol groups binding almost irreversibly with the surface of gold. Our approach makes it possible to obtain composites without additional modification. It is also applicable to preparing composites with small $\mathrm{Au}$ nanospheres, when diblock copolymers are introduced into their shell directly during their synthesis.

\section{EXPERIMENTAL}

\section{Reagents and Equipment}

For the synthesis of gold nanoparticles, aurichlorehydric acid $\left(\mathrm{HAuCl}_{4} \cdot n \mathrm{H}_{2} \mathrm{O}\right.$, reagent grade, 49 wt \% $\mathrm{Au}$, Aurat, Moscow), hexadecyltrimethylammonium bromide (CTAB, 99\%, Sigma), tetraoctylammonium bromide (TOAB, 98\%, Acros Organics), sodium borohydride $\left(\mathrm{NaBH}_{4}, 99 \%\right.$, Acros Organics), Lascorbic acid (99\%, Sigma), and silver nitrate $\left(\mathrm{AgNO}_{3}, 99.9 \%\right.$, Aldrich) were used.

Nanocomposites were obtained from diblock copolymers PS-P-2-VP $\left(M_{\mathrm{n}}=380\right.$ and $156 \mathrm{~kg} / \mathrm{mol}$, $N_{\mathrm{S}}=3650, N_{2-\mathrm{VP}}=1480, \nexists=1.23$ for the entire chain) and PS-P-4-VP $\left(M_{\mathrm{n}}=330\right.$ and $125 \mathrm{~kg} / \mathrm{mol}, N_{\mathrm{S}}=$ $3170, N_{4-\mathrm{VP}}=1190, \nexists=1.18$ for the entire chain) produced by Polymer Source (Canada). On the basis of the composition of the copolymers, P-2-VP and P-4VP blocks should form cylindrical micelles in the microphase-separated state and PS blocks should form a continuum surrounding them.

Solvents (toluene, chloroform, and dioxane) were distilled prior to use. All glassware contacting $\mathrm{HAuCl}_{4}$ was preliminarily washed with aqua regia and deionized water. Silicon substrates used for casting thin films were washed with a hot mixture of concentrated ammonium hydroxide and hydrogen peroxide taken at a ratio of $1: 1$ (take care: it vigorously reacts with organic substances) and deionized water and then dried in the flow of argon. Immediately prior to film casting, the substrates were treated with a low-temperature $\mathrm{H}+\mathrm{O}_{2}$ plasma on a Solarus setup (Gatan, United States).

The thermomechanical properties of block copolymers and related composites were characterized by DSC, DMA, and TGA on DSC $823 \mathrm{e} / 400$, DMA/STDA 861e, and TGA/DSC 1 (MettlerToledo, Switzerland) equipment, respectively.

Scanning electron microscopic (SEM) images of composite films were obtained on a Merlin electron microscope (Carl Zeiss, Germany) using detectors of secondary and backscattered electrons. All films were dried in vacuum prior to measurements. Transmission electron microscopic (TEM) images were obtained on a LEO 912AB-Omega electron microscope (Carl Zeiss, Germany) at an accelerating voltage of $100 \mathrm{kV}$. Samples were prepared by drying sol droplets on a copper grid coated with the polyvinyl formal film. The length and diameter of nanorods were measured by means of open-source software ImageJ 1.50b (Wayne Rasband, United States).

\section{Synthesis of Gold Nanoparticles with a Polymer Shell}

Spherical gold nanoparticles with polymer shells were synthesized via a modified method [32]. The aqueous solution of $\mathrm{HAuCl}_{4}(0.762 \mathrm{mmol}$ in $25.4 \mathrm{~mL})$ was mixed with the toluene solution of TOAB $(15.24 \mathrm{mmol}$ in $423 \mathrm{~mL})$. After $30 \mathrm{~min}$ of vigorous stirring, gold was completely transferred to the organic phase and the aqueous phase was removed. A freshly prepared aqueous solution of $\mathrm{NaBH}_{4}(8.5 \mathrm{mmol}$ in $21.3 \mathrm{~mL}$ ) was added under intense stirring by means of a dropping funnel at a rate of one drop $(\approx 30 \mu \mathrm{L})$ every $15 \mathrm{~s}$. After a while, the solution bleached out and then began to darken. At this moment, the remaining solution of the reducing agent was added, and the reaction mass was stirred for $24 \mathrm{~h}$. Afterwards, the aqueous phase was removed, and a polymer stabilizer was introduced into the organic phase in a 19 -fold excess (by weight) with respect to gold nanoparticles. The block copolymer PS-P-2-VP was added as a toluene solution at $40^{\circ} \mathrm{C}$, while the block copolymer PS P 4 VP was added as a chloroform solution at room temperature. After polymer addition, the sol was stirred 

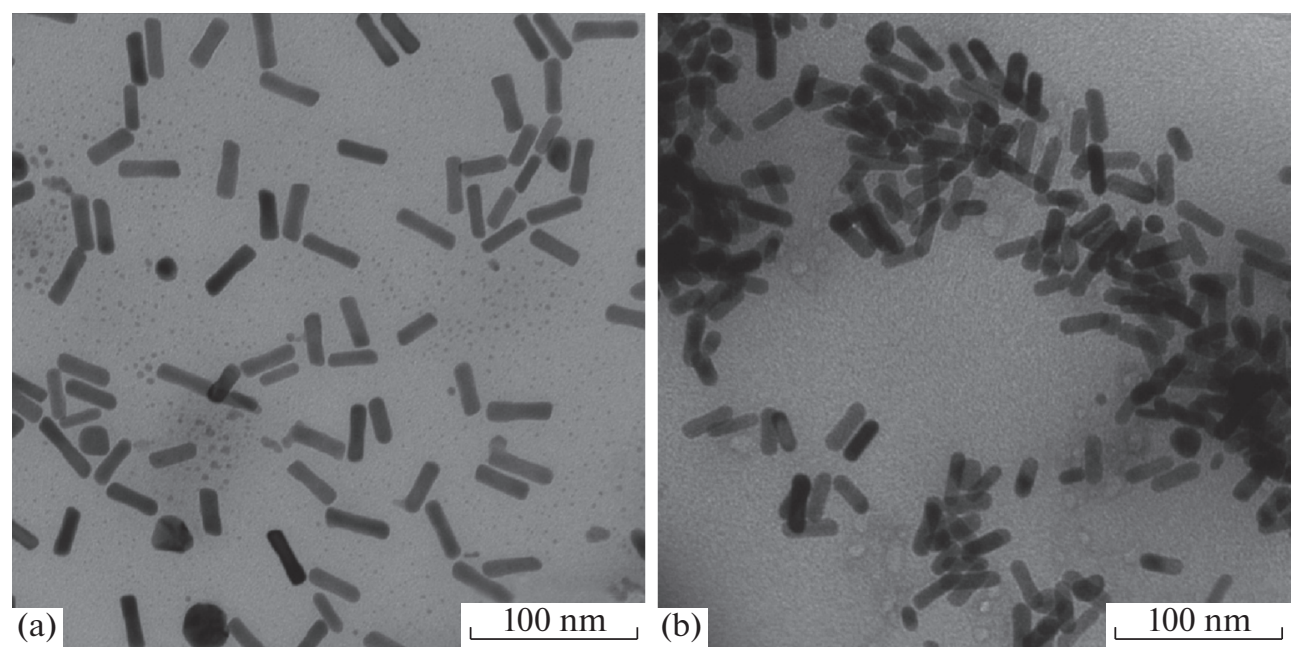

Fig. 1. TEM images of the sol of Au nanorods (a) before and (b) after phase transfer from aqueous medium to chloroform. Stabilizers are (a) CTAB and (b) PS-P-2-VP [28].

for three days. Afterwards, the sol was evaporated to dryness, dispersed in the minimum quantity of chloroform, and precipitated with a 15 -fold excess of ethanol. The precipitate was centrifuged for $20 \mathrm{~min}$ at a rotation speed of $6000 \mathrm{rpm}$. Reprecipitation with the subsequent centrifugation was repeated, and nanoparticles were redispersed in chloroform. Yield was $95 \%$.

According to the TGA data, the as-obtained composites contained $5 \mathrm{wt} \%$ of Au nanoparticles. Composites with $1 \mathrm{wt} \%$ of Au nanoparticles were obtained via addition of PS-P-2-VP or PS-P-4-VP chloroform solutions. After stirring for one day, they were briefly treated in an ultrasound bath and evaporated to dryness in a flow of nitrogen. Finally, chloroform was again added to prepare solutions with a concentration of $0.6-0.9 \mathrm{wt} \%$.

\section{Synthesis of Gold Nanorods}

Au nanorods with polymer shells were synthesized via the modified methodology $[26,27,33]$. Initially, a seeding solution was prepared via addition of $0.1 \mathrm{~mL}$ of $0.01 \mathrm{M} \mathrm{HAuCl}_{4}$ aqueous solution to $3.0 \mathrm{~mL}$ of $0.1 \mathrm{M}$ $\mathrm{CTAB}$ aqueous solution under stirring. When the medium acquired a bright yellow-brown color, $0.24 \mathrm{~mL}$ of $0.01 \mathrm{M}$ ice aqueous solution of sodium borohydride was quickly added under vigorous stirring. The soaking time for seeds was $10 \mathrm{~min}$. During this period, solutions for the synthesis of rods were prepared. For this purpose, $31.7 \mathrm{~mL}$ of $0.001 \mathrm{M} \mathrm{HAu-}$ $\mathrm{Cl}_{4}$ aqueous solution and $3.17 \mathrm{~mL}$ of $0.004 \mathrm{M} \mathrm{AgNO}_{3}$ aqueous solution were added to $63.4 \mathrm{~mL}$ of $0.2 \mathrm{M}$ CTAB aqueous solution. Then, $0.66 \mathrm{~mL}$ of $0.08 \mathrm{M}$ aqueous ascorbic acid was added and the solution was thoroughly stirred until discoloration. Just after that, $0.443 \mathrm{~mL}$ of the solution of seeds was introduced. After a short-term stirring, the system was left for three hours at $30^{\circ} \mathrm{C}$. The obtained deep crimson solution was placed in a fridge for a day. Afterwards, the precipitated CTAB was separated by centrifugation at $6000 \mathrm{rpm}$ for $3 \mathrm{~min}$. The resulting solution was heated to $30^{\circ} \mathrm{C}$ and centrifuged at $11000 \mathrm{rpm}$ for one hour; the precipitate was redispersed in the initial amount of water. The centrifugation-redispersion cycle was repeated three times, wherein for the third time, the precipitate was redispersed in $1.1 \mathrm{~mL}$ of water. The obtained concentrated sol of Au nanorods (Fig. 1a) was used for introduction into polymer solutions. The yield amounted to $70-90 \%$, and the fraction of nanorods exceeded $90 \%$.

\section{Introduction of Nanorods into Block Copolymers $P S-P-2-V P$ and $P S-P-4-V P$}

Copolymer PS-P-4-VP $(7.0 \mathrm{mg})$ was dissolved in a mixture of THF $(1.6 \mathrm{~mL})$ and ethanol $(0.7 \mathrm{~mL})$ or PSP 2 VP $(5.8 \mathrm{mg})$ was dissolved in THF $(1.9 \mathrm{~mL})$. Then, $81 \mu \mathrm{L}$ of the concentrated sol of nanorods in PS-P-4-VP (or $67 \mu \mathrm{L}$ in PS-P-2-VP) were intreduced under vigorous stirring at a rate of at most $1 \mu \mathrm{L} / \mathrm{s}$. Afterwards, the stirring speed was decreased and the solution was left overnight. The resulting solution was placed in an ultrasound bath for $30 \mathrm{~min}$ and dried in vacuum. The as-obtained sol of nanorods was dissolved in chloroform (Fig. 1b). The yield was 50\%, the average length of nanorods was $30 \mathrm{~nm}$, and their average diameter was $9 \mathrm{~nm}$.

\section{Preparation of Composite Thin Films}

Films were cast using a WS-650Mz-23NPP spin coater (Laurell, United States. Composite solutions in chloroform $(60-70 \mu \mathrm{L}, 0.6-0.9 \mathrm{wt} \%)$ were cast on a silicon substrate, and the substrate was rotated at a speed of $3000 \mathrm{rpm}$. In order to obtain the structure of 
Table 1. Characteristics of the synthesized composites

\begin{tabular}{l|l|l|c|c|c}
\hline \multicolumn{1}{c|}{ Matrix } & $\begin{array}{c}\text { Stabilizer } \\
\text { of nanoparticles }\end{array}$ & \multicolumn{1}{|c|}{$\begin{array}{c}\text { Shape } \\
\text { of nanoparticles }\end{array}$} & $\begin{array}{c}\text { Concentration } \\
\text { of nanoparticles, } \\
\text { wt \% (TGA) }\end{array}$ & $\begin{array}{c}\text { SEM image } \\
\text { in the figure }\end{array}$ \\
\hline PS-P-2-VP & PS-P-2-VP & Spheres & 5 & 1 & $3 \mathrm{a}, 4 \mathrm{a}$ \\
PS-P-2-VP & PS-P-2-VP & Spheres & 5 & 5 & $3 \mathrm{~b}$ \\
PS-P-2-VP & PS-P-4-VP & Spheres & 5 & 1 & $4 \mathrm{~b}$ \\
PS-P-4-VP & PS-P-4-VP & Spheres & 5 & 5 & $2 \mathrm{a}, 2 \mathrm{~b}$ \\
PS-P-4-VP & PS-P-4-VP & Rods & $9 \times 30$ & 5 & $6 \mathrm{a}$ \\
PS-P-4-VP & PS-P-2-VP & Rods & $9 \times 30$ & 5 & $6 \mathrm{~b}$ \\
\hline
\end{tabular}

vertical cylinders, the prepared films were treated for one to two hours in dioxane vapors [34]. The contrast of polyvinylpyridine domains on SEM images was increased via treatment of PS-P-4-VP-based composite films with molecular iodine vapors for one hour and dried in vacuum to remove free iodine.

\section{RESULTS AND DISCUSSION}

The experimental section describes the method of using styrene-vinylpyridine block copolymers to stabilize gold nanoparticles of different sizes and shapes. We managed to obtain organodisperse spherical gold nanoparticles with a diameter of $\sim 5 \mathrm{~nm}$ with polymer shells by means of a well-established two-phase method [32] via replacement of the low-molecularmass stabilizer TOAB with a block copolymer. For the initially aqueous-dispersed nanorods of larger sizes $(9 \times 30 \mathrm{~nm})$ stabilized by CTAB, the block copolymers were used as transfer agents of nanorods to the organic phase. Au nanoparticles with copolymers PS-P-2-VP or PS-P-4-VP in the shell proved to be well dispersible in organic solvents. On the basis of these copolymers, a number of composites with nanoparticle concentra- tions of 1 and $5 \mathrm{wt} \%$ (according to TGA) were obtained; their characteristics are presented in Table 1. Structural investigations of microphase-separated thin films revealed the distribution of nanoparticles in them. With this aim in view, the SEM images of composite films with spherical and rodlike nanoparticles and DSC thermograms describing the effect of nanoparticles on the glass-transition temperature of individual blocks of the copolymers were obtained. The results will be discussed below.

In all cases, gold nanoparticles are arranged primarily in cylindrical domains formed by P-2-VP or P 4-VP. This effect is especially pronounced when images of composite films are compared before (Fig. 2a) and after (Fig. 2b) their coloration with iodine vapor forming a complex with pyridine rings in nonpolar media [35]. Gold nanoparticles, which are clearly seen in Fig. $2 \mathrm{a}$ as bright spots, are absent, with few exceptions, in Fig. 2b. Consequently, both gold and iodine are localized in the same type of domains, namely, P-4-VP.

The selectivity of Au nanoparticles with respect to domains of P-2-VP and P-4-VP in the styrene-based copolymers $[24,30,36,37]$ is also explained by the
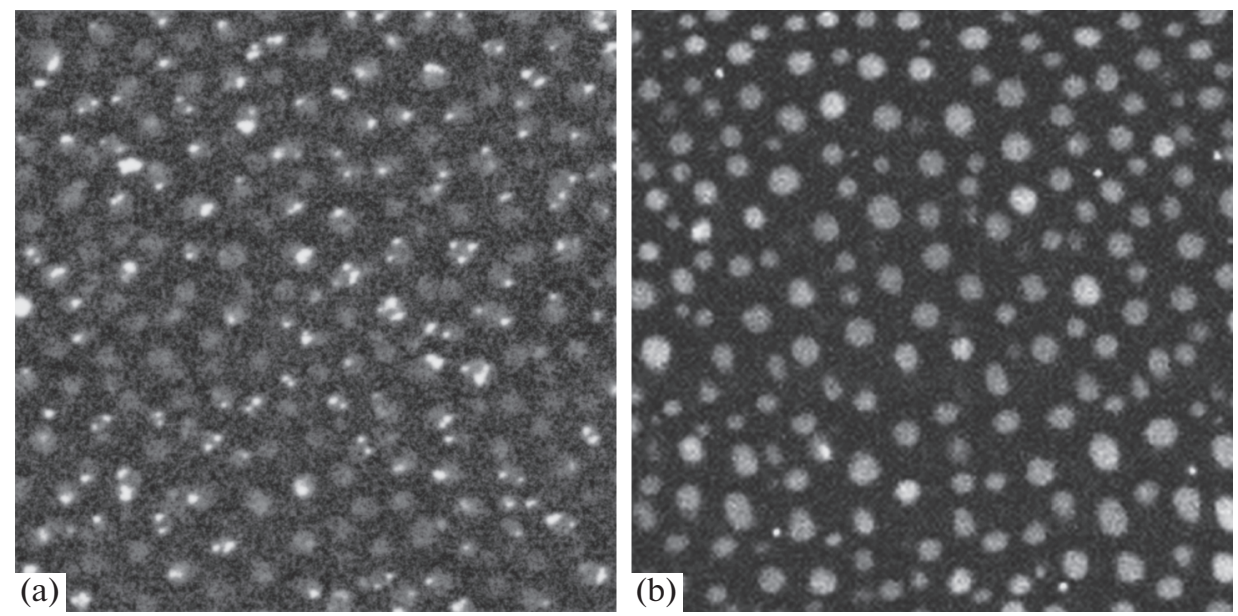

Fig. 2. SEM images $(1 \times 1 \mu \mathrm{m})$ of the microphase-separated film based on the PS-P-4-VP composite containing 5 wt $\%$ of spherical Au nanoparticles (a) before and (b) after in iodine vapor. 

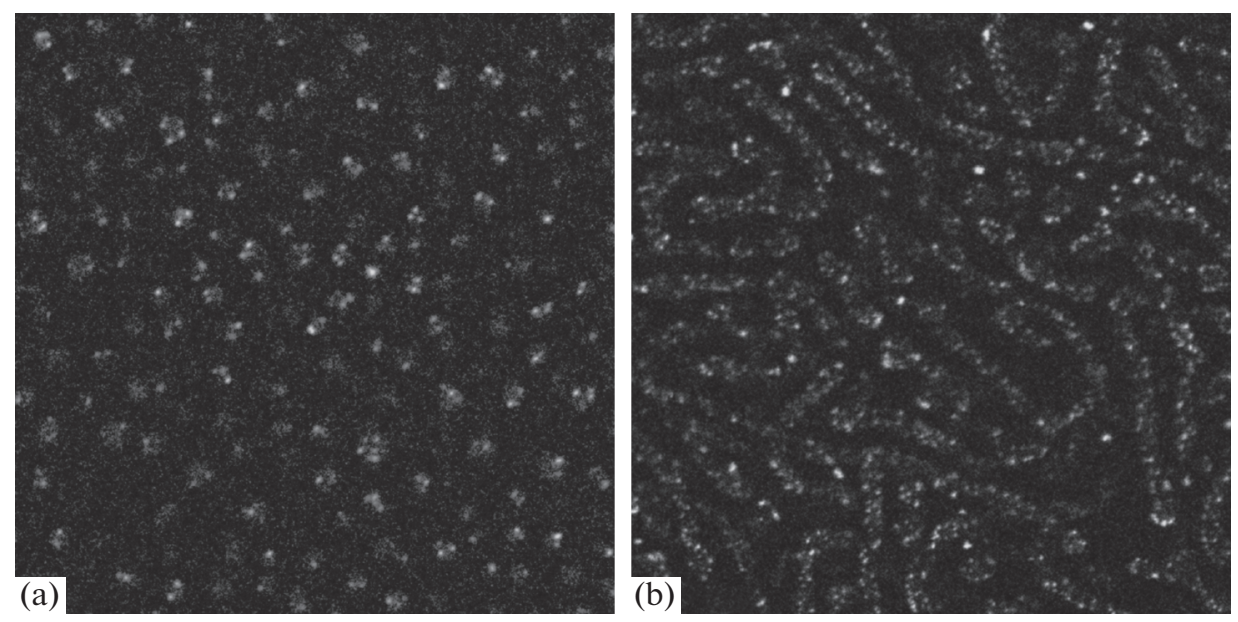

Fig. 3. SEM images $(2 \times 2 \mu \mathrm{m})$ of the microphase-separated films based on the PS-P-2-VP composite containing (a) 1 and (b) 5 wt $\%$ of spherical Au nanoparticles.

interaction of nanoparticles with pyridine, which is mainly contributed from the lone electron pair of the nitrogen atom [38]. It should be noted that, before the present work, the introduction of nanoparticles into copolymers was possible only via either the reduction of atrichlorehydric acid directly in a polymer matrix $[36,37]$ or the use of thiol-terminated P-2-VP [24, 30]. The former method provides a way to synthesize only small (less than $5 \mathrm{~nm}$ ) spheres, while the latter requires introduction of an additional strongly interacting component into the composite.

The use of high-molecular-mass copolymers made it possible to show for the first time that, although spherical nanoparticles are arranged within $\mathrm{P}-2-\mathrm{VP}$ or P-4-VP domains, they are localized near domain boundaries (Figs. 2-4). This behavior may be explained by presence of the pyridine ring in each repeating unit of the vinylpyridine block, promoting the "winding" of these blocks on a particle and their effective shortening. Therefore, the localization of nanoparticles in the middle of cylindrical domains could result in the energetically unfavorable pulling of styrene blocks into the domains of vinylpyridine. It would be of interest to study this effect in more detail using copolymers with different molecular masses of the blocks.

The arrangement of nanoparticles near the domain boundary of cylinders is undoubtedly related to the appearance of only one glass-transition temperature on the thermogram of the composite based on the PSP-4-VP copolymer (Fig. 5) consisting of blocks with substantially different glass-transition temperatures $\left(73^{\circ} \mathrm{C}\right.$ for PS and $141^{\circ} \mathrm{C}$ for $\left.\mathrm{P}-4-\mathrm{VP}\right)$. This behavior is characteristic of materials in which the filler plays the role of a surfactant decreasing the surface tension between domains. Note that the effect of a noticeable reduction in $T_{\mathrm{g}}$ for $\mathrm{P}-4-\mathrm{VP}$ blocks in the presence of Au nanoparticles was described [36] and explained, as a rule, by a decrease in the polymer density near the surface of particles. However, in the case of lamellar morphology, the DSC data [36] still indicate that the $T_{\mathrm{g}}$ of PS blocks containing almost no nanoparticles is lower.

For the copolymer PS-P-2-VP, both in its pure form and in the presence of 5\% nanoparticles, only one $T_{\mathrm{g}}=99^{\circ} \mathrm{C}$ is determined, since the glass-transition temperatures of PS and PS 2 VP homopolymers differ insignificantly. It may be concluded that the introduction of nanoparticles reduces the difference between characteristics of the composites based on PS-P-2-VP and PS-P-4-VP copolymers. Both copolymers stabilize well nanoparticles, while a slight difference in the molecular masses of vinylpyridine blocks ( $125 \mathrm{~kg} / \mathrm{mol}$ for P-4-VP and $156 \mathrm{~kg} / \mathrm{mol}$ for PZ-VP) causes a somewhat lower diameter of cylindrical domains in the case of P-4-VP. Moreover, despite the thermodynamic incompatibility of PS-P-2-VP and PS-P-4-VP blocks [39], the composite, in which spherical nanoparticles $(1 \mathrm{wt} \%)$ are initially stabilized by the PS-P-4-VP copolymer and afterwards are placed in the matrix of the PS-P-2-VP copolymer (Fig. 4b), demonstrates the same but a slightly more polydisperse microstructure of vertical cylinders as the composite in Fig. 4a, in which has the polymer component consists of PS-P-2-VP solely. The distribution of nanoparticles in the matrix in both cases is identical.

According to much larger sizes of nanorods, it can assumed that the "winding" of the copolymer on them should be even more pronounced than that in the case of spherical nanoparticles. Geometrically, the arrangement of nanorods near the domain boundary requires their orientation along the axis of cylinders. However, as is seen in Fig. 6, a larger part of nanorods are arranged in the film plane rather than vertically. Note that a similar effect was observed in [24]; using 

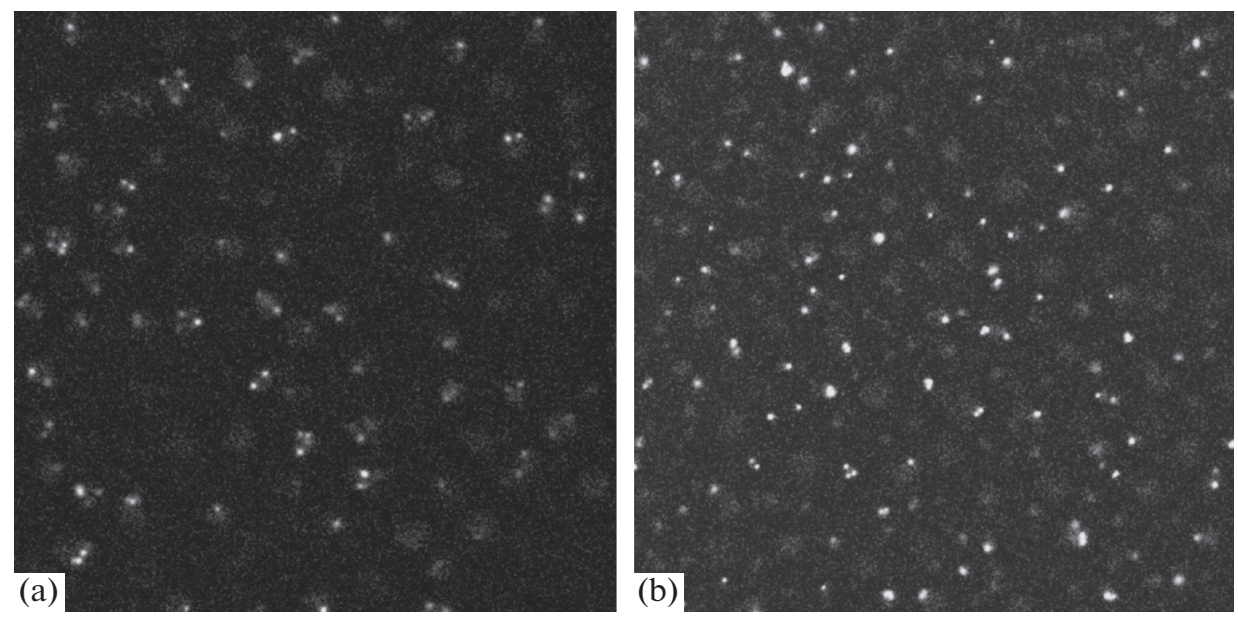

Fig. 4. SEM images $(1.5 \times 1.5 \mu \mathrm{m})$ of the microphase-separated films based on the PS-P-2-VP composite containing $1 \mathrm{wt} \%$ of spherical Au nanoparticles with (a) PS-P-2-VP and (b) PS-P-4-VP in the shell.

the method of layer-by-layer film etching, it was shown that a significant part of rods in P-2-VP domains are localized near the substrate, where cylinders themselves are oriented horizontally rather than vertically. However, in such a case, the position of nanorods in the film on SEM images could not correlate strongly with the arrangement of cylindrical domains on its free surface. Another possible reason behind the difference in the arrangements of spherical nanoparticles and nanorods in our case may be different rates of the replacement of the low-molecularmass stabilizer by block copolymers (the process lasts

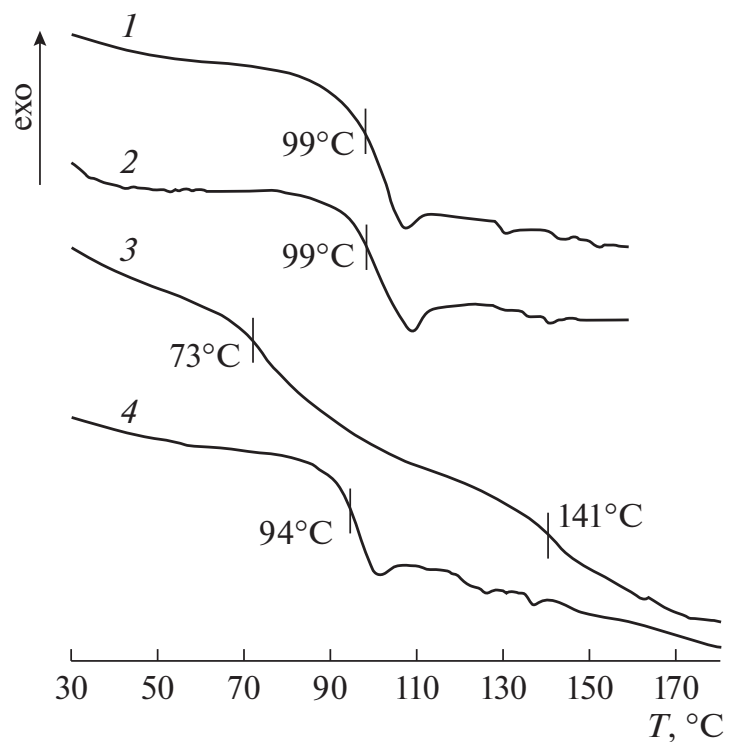

Fig. 5. DSC curves of the second heating for block copolymers (1) PS-P-2-VP and (3) PS-P-4-VP and (2, 4) their composites with $5 \mathrm{wt} \%$ of spherical Au nanoparticles Au. Numbers on the curves the glass-transition temperatures. for $\sim 1$ day for spherical nanoparticles and only $\sim 1 \mathrm{~min}$ for nanorods); as a result, nanorods are bound to a random and relatively short part of the vinylpyridine block).

The structures of composites based on PS-P-2-VP, PS-P-4-VP, and P-2-VP-P-4-VP block copolymers were compared at different contents of Au nanoparticles [37]. It was found that the nanoparticles are the most selective with respect to $\mathrm{P}-4-\mathrm{VP}$ blocks. This finding may be explained by a higher accessibility of nitrogen atom at position 4 of the pyridine ring compared with position 2 . In the latter case, the insertion of nanoparticles requires a considerable change in the conformation of the corresponding blocks; therefore, the size of $\mathrm{P}-2-\mathrm{VP}$ domains increases to a more considerable extent compared to that of $\mathrm{P}-4-\mathrm{VP}$ domains. In our system, distributions of $1 \%$ of spherical nanoparticles and $5 \%$ of nanorods in the microstructure of vertical cylinders formed by PS-P-2-VP and PS-P-4-VP copolymers are qualitatively similar (Figs. 2, 3a, 4a, 6), but for composites containing 5\% of nanospheres, a mixed microstructure of horizontal and vertical cylinders arises (Fig. 3b). Under fixed conditions at boundaries of the thin film, the type of formed morphology is usually determined by the ratio between the film thickness and the domain size. Since all films were cast using the same experimental procedure, the swelling of P-2-VP domains upon the introduction of $5 \%$ of nanoparticles into them gave rise to a noticeable change in this ratio; as a result, the morphology can change qualitatively.

It should be noted that appearance of the mixed structure visualized in Fig. 3b clearly demonstrates the uniform distribution of spherical nanoparticles in $\mathbf{P}_{2}$ VP domains. In composite films with the vertical orientation of domains, this conclusion requires a more complicated TEM method appropriate for transversally ultratomed films. 

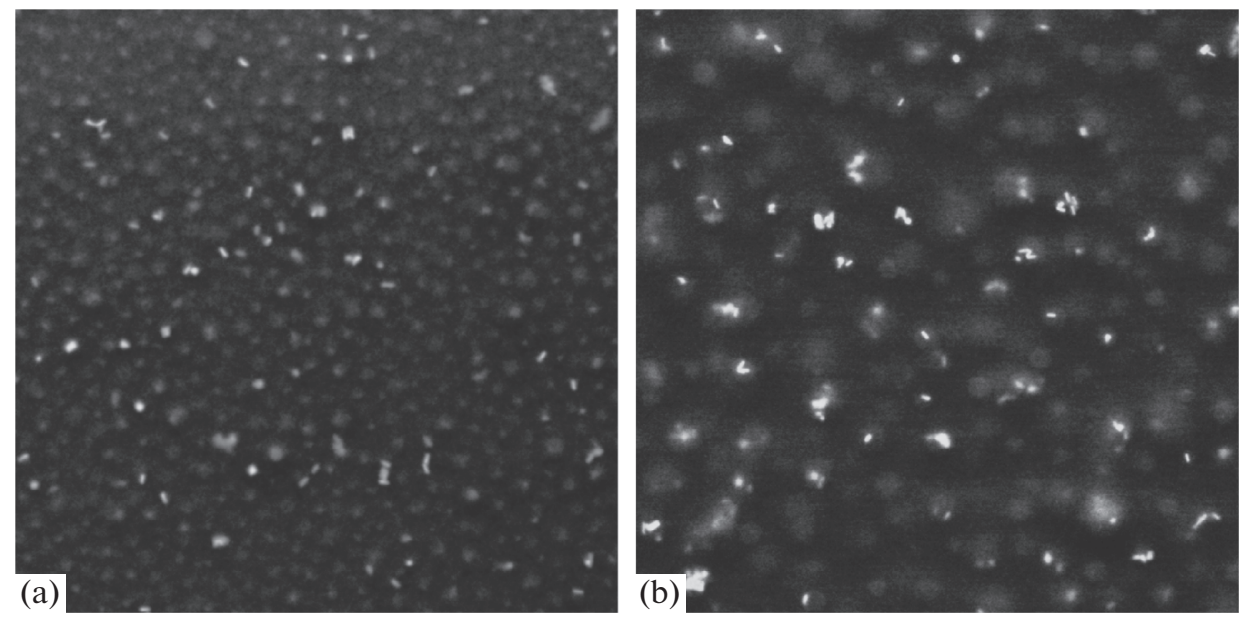

Fig. 6. SEM images $(2 \times 2 \mu \mathrm{m})$ of the microphase-separated films based on the block copolymers (a) PS-P-4-VP and (b) PS-P2-VP containing 5 wt $\%$ of Au nanorods.

Thus, a novel approach to the preparation of composites based on styrene-vinylpyridine block copolymers containing isotropic and anisotropic gold nanoparticles has been proposed. A specific feature of the approach is that nanoparticles are stabilized directly by the matrix polymer in the absence of any additives. The selectivity of Au nanoparticles with respect to vinylpyridine blocks makes it possible to reach a spatially modulated density distribution of $\mathrm{Au}$ nanoparticles reproducing the microphase-separated matrix block copolymer structure. The synthesized composites possess a stable microstructure preventing the aggregation of nanoparticles upon multiple precipitation-drying-redispersion cycles and under high-temperature conditions, as was shown in [28].

\section{ACKNOWLEDGMENTS}

We are grateful to S.S. Abramchuk (Institute of Organoelement Compounds, Russian Academy of Sciences) for his help with TEM measurements on the equipment from the Center for Collective Use Transmission Electron Microscopy at Moscow State University.

This work was supported by the Russian Science Foundation, project no. 16-13-10280.

\section{REFERENCES}

1. C. M. Bates and F. S. Bates, Macromolecules 50 (3), 3 (2017).

2. M. Luo and T. H. Epps III, Macromolecules 46 (19), 7567 (2013).

3. H. Hu, M. Gopinadhan, and C. O. Osuji, Soft Matter 10 (22), 3867 (2014).

4. H. Feng, X. Lu, W. Wang, N. -G. Kang, J. W. Mays, Polymers 9 (10), 494 (2017).
5. C. M. Bates, M. J. Maher, D. W. Janes, C. J. Ellison, and C. G. Willson, Macromolecules 47 (1), 2 (2014).

6. P. D. Topham, A. J. Parnell, and R. C. Hiorns, J. Polym. Sci., Part B: Polym. Phys. 49 (16), 1131 (2011).

7. V. Abetz, Macromol. Rapid Commun. 36 (1), 10 (2015).

8. HT. H. Epps and R. K. O' Reilly, Chem. Sci. 7 (3), 1674 (2016).

9. M. R. Bockstaller, R. A. Mickiewicz, and E. L. Thomas, Adv. Mater. 17 (11), 1331 (2005).

10. T. N. Hoheisel, K. Hur, and U. B. Wiesner, Prog. Polym. Sci. 40, 3 (2015).

11. B. Sarkar and P. Alexandridis, Prog. Polym. Sci. 40, 33 (2015).

12. M. A. Boles, M. Engel, and D. V. Talapin, Chem. Rev. 116 (18), 11220 (2016).

13. A. C. Balazs, T. Emrick, and T. P. Russell, Science 314 (5802), 1107 (2006).

14. V. A. Gerasin, E. M. Antipov, V. V. Karbushev, V. G. Kulichikhin, G. P. Karpacheva, R. V. Talroze, and Y. V. Kudryavtsev, Russ. Chem. Rev. 82 (4), 303 (2013).

15. B. Gao, M. J. Rozin, and A. R. Tao, Nanoscale 5 (13), 5677 (2013).

16. C. Saldias, S. Bonardd, C. Quezada, D. Radic, and A. Leiva, J. Nanosci. Nanotechnol. 17 (1), 87.

17. M. A. Osipov and M. V. Gorkunov, Eur. Phys. J. E: Soft Matter Biol. Phys. 39 (12), 126 (2016).

18. M. A. Osipov, A. S. Ushakova, and M. V. Gorkunov, Liq. Cryst. 44 (12-13), 1861 (2017).

19. M. A. Osipov and A. S. Ushakova, J. Mol. Liq. (2018). doi: 10.1016/j.molliq.2018.01.048

20. A. V. Berezkin, Y. V. Kudryavtsev, M. V. Gorkunov, and M. A. Osipov, J. Chem. Phys. 146 (14), 144902 (2017).

21. R. D. Deshmukh, Y. Liu, and R. J. Composto, Nano Lett. 7 (12), 3662 (2007). 
22. W. Li, P. Zhang, M. Dai, J. He, T. Babu, Y.J-L. Xu, R. Deng, R. Liang, M. -H. Lu, Z. Nie, and J. Zhu, Macromolecules 46 (6), 2241 (2013).

23. Z. C. Liu, H. Y. Huang, and T. B. He, Small 9 (4), 505 (2013).

24. B. Rasin, H. Chao, G. Jiang, D. Wang, R. A. Riggleman, and R. J. Composto, Soft Matter 12 (7), 2177 (2016).

25. N. Li, P. Zhao, and D. Astruc, Angew. Chem., Int. Ed. Engl. 53 (7), 1756 (2014).

26. B. Nikoobakht and M. A. El-Sayed, Chem. Mater. 15 (10), 1957 (2003).

27. T. K. Sau and C. J. Murphy, Langmuir 20 (15), 6414 (2004).

28. Y. I. Derikov, G. A. Shandryuk, R. V. Talroze, A. A. Ezhov, and Y. V. Kudryavtsev, Beilstein J. Nanotechnol. 9, 616 (2018).

29. A. Roescher and M. Möller, Adv. Mater. 7 (2), 151 (1995).

30. B. J. Kim, J. Bang, C. Hawker, and E. J. Kramer, Macromolecules 39 (12), 4108 (2006).
31. Z. Fan, M. Köhn Serrano, S. Agarwal, and A. Greiner, Adv. Mater. 27 (26), 3888 (2015).

32. A. E. Saunders, M. B. Sigman, Jr., and B. A. Korgel, J. Phys. Chem. B 108 (1), 193 (2004).

33. M. R. K. Ali, B. Snyder, and M. A. El-Sayed, Langmuir 28 (25), 9807 (2012).

34. E. B. Gowd, T. Koga, M. K. Endoh, K. Kumar, and M. Stamm, Soft Matter 10 (39), 7753 (2014).

35. E. K. Plyler and R. S. Mulliken, J. Am. Chem. Soc 81 (4), 823 (1959).

36. C. Mendoza, T. Pietsch, J. S. Gutmann, D. Jehnichen, N. Gindy, and A. Fahmi, Macromolecules 42 (4), 1203 (2009).

37. J. Lee, J. Kwak, C. Hans. H. Choi, and J. K. Kim, Macromolecules 50 (23), 9373 (2017).

38. D. Mollenhauer, J. Floss, H. U. Reissig, E. Voloshina, and B. Paulus, J. Comput. Chem. 32 (9), 1839 (2011).

39. S. H. Han, D. H. Lee, and J. K. Kim, Macromolecules 40 (20), 7416 (2007).

Translated by A. Yakimansky 